Rev. Elev. Méd. vét. Pays trop., 1974, 27 (4) : 467-473

\title{
Modifications de certains constituants biochimiques du sang chez les zébus naturellement infestés par Fasciola gigantica
}

\author{
par J. BLANCOU $(*)$, Y. CHENEAU $\left({ }^{*}\right)$ et A. BOUCHET (*) \\ (avec la cotlaboration technique de Françoise ALEXANDRE et D. RAMBELOMANANA)
}

RESUME

Le dosage de douze constituants biochimiques du sang de cinquante zébus atteints de fasciolose grave démontre que le taux de six d'entre eux varie, de façon significative ou hautement significative, par rapport à celui relevé chez cinquante témoins.

Il s'agit des taux d'albumine, de bilirubine, de transaminase glutamique-pyruvique, de gamma-globulines, de Béta-globulines et des protéines sériques. Ce sont les variations des taux de bilirubine et de transaminase glutamique-pyruvique qui semblent en corrélation la plus étroite avec la fasciolose et peuvent confırmer le diagnostic de cette parasitose.

\section{INTRODUCTION}

L'étude des modifications des constituants biochimiques essentiels du sang dans la fasciolose a été surtout réalisée chez les ovins infestés artificiellement $(2,3,7,13,15,16,17,22$, 24, 26).

Les études de même ordre sont beaucoup plus fragmentaires chez les bovins et concernent uniquement le parasitisme par Fasciola hepatica $(1,6,8,9,10,12,14,18,20,21,27)$, le plus souvent créé artificiellement.

Nous nous sommes donc proposé, dans le présent travail, de contribuer à élargir nos connaissances sur la physio-pathologie de l'infestation parasitaire naturelle, à Fasciola gigantica en particulier. Notre but était aussi de dégager, éventuellement, des critères de diagnostic des états avancés de fasciolose, complémentaire des méthodes coproscopiques ou immunologiques $(4,5)$.

(*) I.E.M.V.T., Laboratoire central de l'Elevage, B.P. 4, Tananarive, République Malgache.

\section{MATERIEL}

\section{Animaux}

Les sangs des 100 animaux objets des analyses biochimiques que nous rapportons ont été choisis parmi ceux de 1770 zébus malgaches tués dans un abattoir industriel de Tananarive, au cours des mois de novembre-décembre et janvier 1972-1973. Ce sont des animaux âgés de 6 ans ou plus.

Etant donné l'incertitude quant à l'existence et la gravité de l'infestation au moment de l'abattage, une trentaine des zébus abattus dans la journée font l'objet d'un prélèvement de sang au moment de la saignée. Ce n'est qu'après le résultat d'autopsie que certains prélèvements sont retenus et groupés en deux catégories.

1. Prélèvements correspondants à des animaux totalement indemnes de lésions macroscopiques de fasciolose ou de tuberculose $\left(^{*}\right)$, qui constituent le groupe témoin.

(*) L'incidence de la tuberculose bovine est de 50 p. 100 sur les sujets abattus à Tananarive. 
2. Prélèvements correspondants à des animaux, exempts de tuberculose, atteints de formes graves de fasciolose : angio-cholite chronique, cirrhose et nombre de douves par foie supérieur à 70 .

\section{Matériel d'analyse}

Matériel classique nécessaire aux prélèvements puis aux analyses effectuées selon les techniques décrites au chapitre suivant.

Certains réactifs sont livrés standardisés et prêts à l'emploi, en particulier pour le dosage des lipides totaux et des transaminases $\left({ }^{*}\right)$.

\section{METHODES}

\section{Prélèvements}

Le sang est prélevé, lors de la saignée jugulaire, en deux flacons de verre: l'un, vide, où sera récolté le sérum; l'autre, contenant du mélange de WINTROBE, où sera récolté le plasma.

\section{Analyses}

Douze tests ont été effectués, choisis parmi ceux qui nous paraissaient avoir le plus de chances d'être modifiés dans les états d'hépatite parasitaire avancée :

\section{Protéinogramme}

Selon la technique de GRASSMAN et HANNIG (électrophorèse sur papier, coloration au noir Amido - $10 \mathrm{~B}$, interprétation par Integraph ELPHOR) (19).

\section{Protéines sériques}

Méthode photométrique au biuret de RIEGLER, technique de GORNALL (11).

\section{Fibrinogène}

Microdosage photométrique par la technique de LECLERC et KHODABANDEH (11).

\section{Lipides totaux}

Méthode pondérale après extraction au méthylal-méthanol par la méthode de DELSAL, technique de HARLEY (11).

\section{Bilirubine totale}

Diazoréaction d'Hijmans VANDENBERGH

$(* *)$ * Mercko-tests ». après libération de la bilirubine totale par la réaction de JENDRASSIK et GROF (11).

Transaminases glutamique oxaloacétique

(T.G.O.) et glutamique pyruvique (T.G.P.)

D'après la technique de FRANKEL et REITMAN (23).

\section{Calcium}

Microdosage par complexométrie (méthodes à la calcéine) selon DREUX et GIRARD (11).

\section{RESULTATS}

Les résultats figurent sur les graphiques suivants, concernant 50 zébus gravement infestés par "Fasciola gigantica " et 50 zébus témoins, indemnes de fasciolose et de tuberculose.

L'analyse statistique des résultats individuels des 100 sujets (FISHER et STUDENT) permet de déterminer les tests les plus discriminants, quant à l'état parasitaire.

Cette relation, nulle pour certains constituants, est au contraire très hautement significative, ou significative, pour d'autres.

La classification, dans l'ordre d'importance du coefficient $« t$ » déterminé entre les deux groupes, parasités et témoins est indiquée dans le tableau p. 471.

\section{DISCUSSION}

En considérant chacun de nos résultats, par rapport à ceux obtenus par d'autres auteurs, nous pouvons encore mieux dégager la valeur qui s'y attache.

\section{Protéinogramme}

Trois de ses composantes varient, dans notre étude, de façon significative ou hautement significative lors de fascioloses graves: Albumines, Bétaglobulines, Gamma Globulines. Cette variation est signalée par la plupart des auteurs précédemment cités. Mais elle n'est pas spécifique de l'hépatite parasitaire et signe seulement un état pathologique infectieux. W. MULLIGAN (21) en donne une explication détaillée dans le cas de la fasciolose, se basant sur l'étude du métabolisme de protéines marquées à l'iode radioactif. L'altération des muqueuses de l'animal parasité au niveau cellulaire entraîne une fuite protéique ( hypoalbuminémie hyperca- 

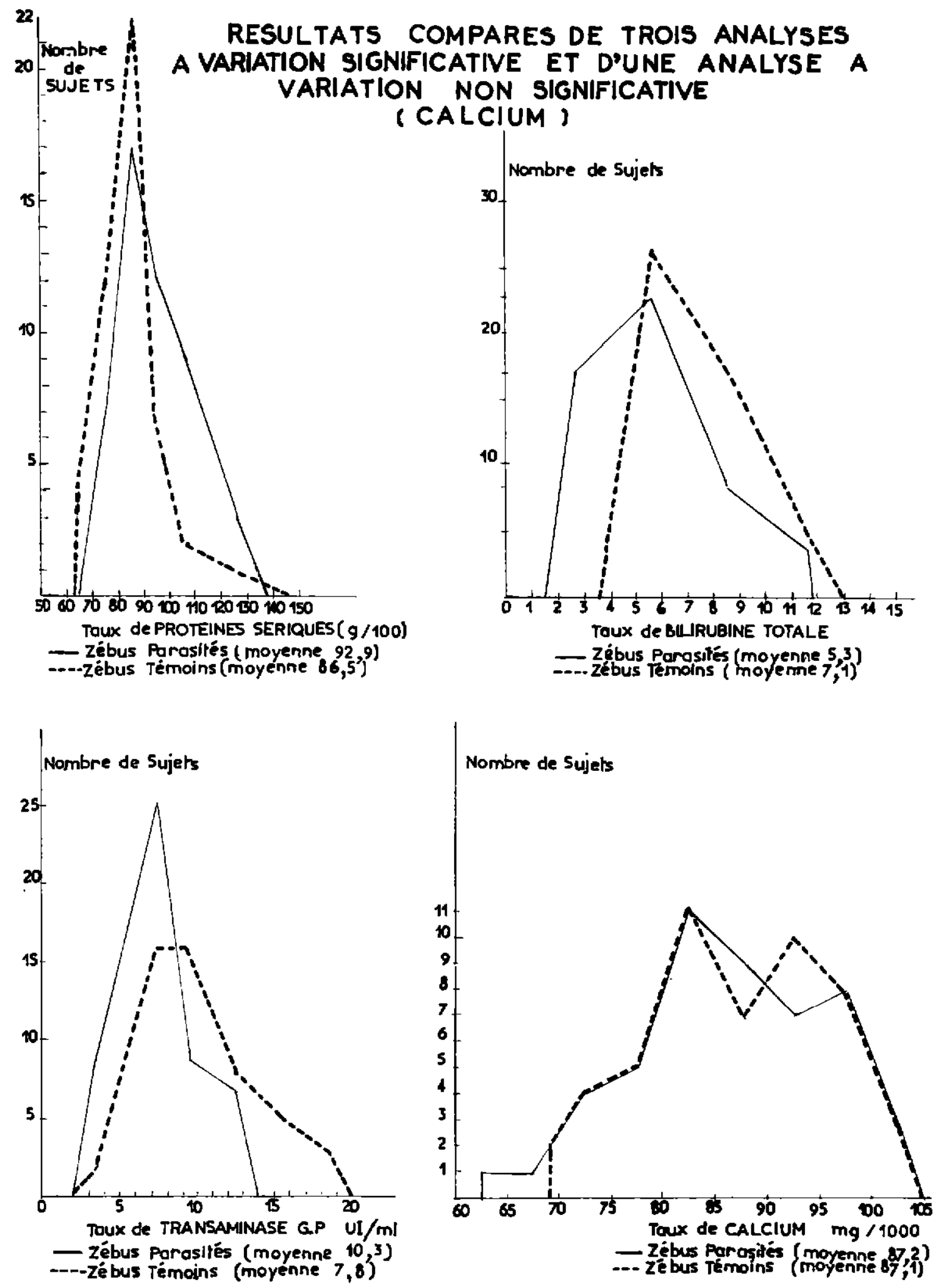


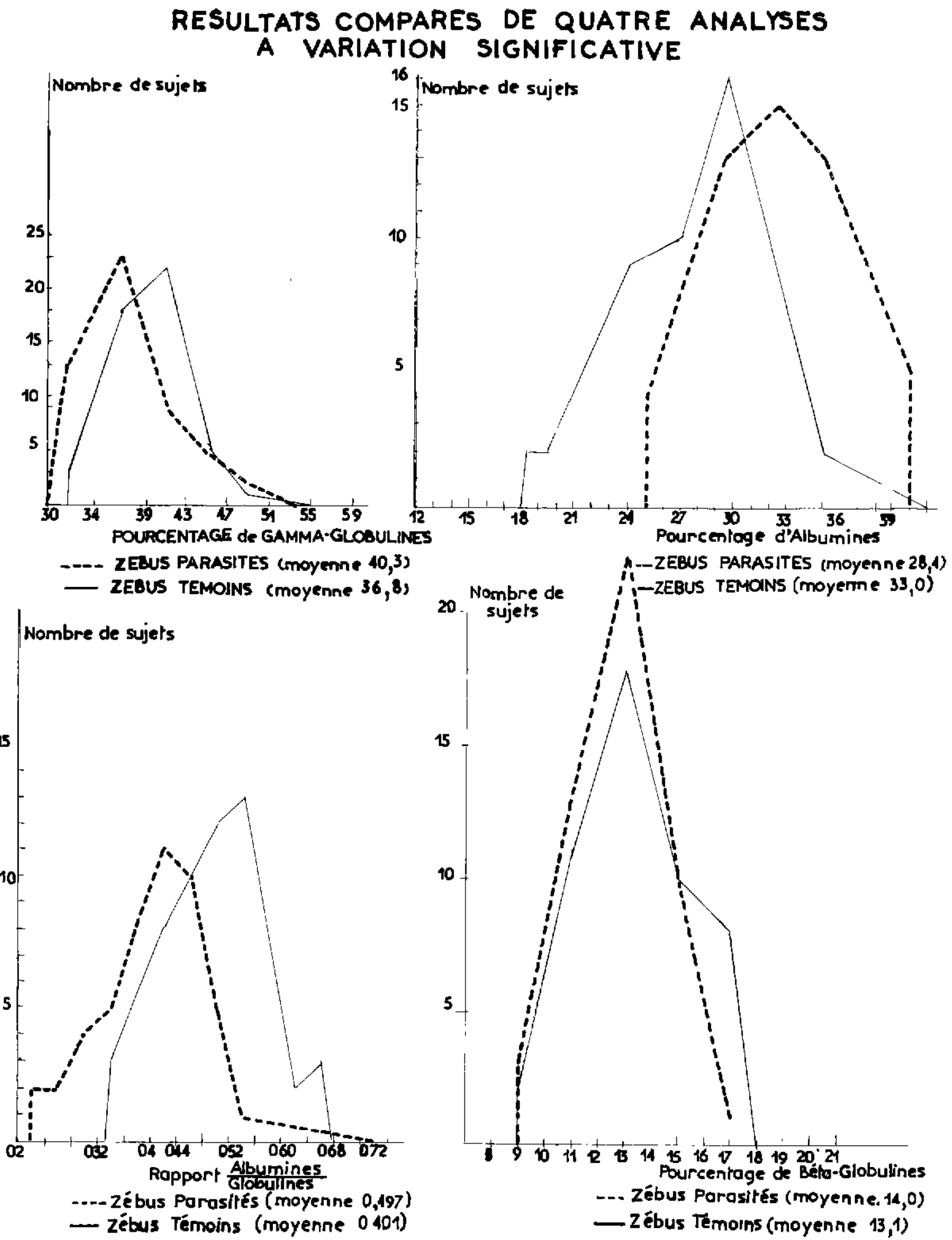




\begin{tabular}{|c|c|c|c|c|}
\hline \multirow{2}{*}{ Analyses } & \multicolumn{2}{|c|}{ Moyennes } & \multirow{2}{*}{ Valeur de $\mathrm{t}^{*}$} & \multirow{2}{*}{ Valeur de la corrélation } \\
\hline & Témoins & Parasités & & \\
\hline Rapport $\frac{\text { Albumines }}{\text { Globulines }}$ & 0,402 & 0,497 & 6,76 & $\begin{array}{l}\text { Hautement significative } \\
\text { (infërieure â } t=0,01 \text { ) }\end{array}$ \\
\hline Albumines & 33 p. 100 & $28,4 \mathrm{p} \cdot 100$ & 5,89 & $\begin{array}{l}\text { Hautement significative } \\
\text { (inférieure à } t=0,01 \text { ) }\end{array}$ \\
\hline Bilirubine & $7,1 \mathrm{mg} / 1000$ & $5,3 \mathrm{mg} / 1000$ & 4,04 & $\begin{array}{l}\text { Hautement significative } \\
\text { (infërieure à } t=0,01 \text { ) }\end{array}$ \\
\hline Gamma globulines & $36,8 \mathrm{p} \cdot 100$ & 40,3 p. 100 & 4,00 & $\begin{array}{l}\text { Hautement significative } \\
\text { (inférieure à } t=0,01 \text { ) }\end{array}$ \\
\hline Transaminases T.G.P. & $7,8 \mathrm{UI} / \mathrm{ml}$ & $10,3 \mathrm{UI} / \mathrm{ml}$ & 3,50 & $\begin{array}{l}\text { Significative } \\
\text { (infërieure à } t=0,01 \text { ) }\end{array}$ \\
\hline Protéines sériques & $86,5 \mathrm{~g} / 100$ & $92,98 / 100$ & 2,32 & $\begin{array}{l}\text { Significative } \\
\text { (inférieure à } \mathrm{t}=0,05 \text { ) }\end{array}$ \\
\hline Bëta glabulines & 13,1 p. 100 & $14 \quad$ p. 100 & 2,11 & $\begin{array}{l}\text { Significative } \\
\text { (inférieure a } t=0,05\end{array}$ \\
\hline Fibrinogène & $7.6 \mathrm{~g} / 1000$ & $8,3 \mathrm{~g} / 1000$ & 1,69 & $\begin{array}{l}\text { Non significative } \\
\text { (infêrieure à } t=0,1\rangle\end{array}$ \\
\hline Transaminases T.G.O. & $63,6 \mathrm{UI} / \mathrm{ml}$ & $55,9 \mathrm{vI} / \mathrm{m} 1$ & 1,55 & $\begin{array}{l}\text { Non significative } \\
\text { (inférieure à } t=0,2 \text { ) }\end{array}$ \\
\hline Lipides totaux & $3,8 \mathrm{~g} / 1000$ & $4,1 \mathrm{~g} / 1000$ & 1,44 & $\begin{array}{l}\text { Non significative } \\
\text { (inférieure à } t=0,2 \text { ) }\end{array}$ \\
\hline Alpha 2 globulines & $10,2 \mathrm{p} \cdot 100$ & $9,8 \mathrm{p} \cdot 100$ & 0,76 & Non significative \\
\hline Alpha 1 globulines & 7 p. 100 & $6,9 \mathrm{p}, 100$ & 0,75 & Non significative \\
\hline Calcium & $87,1 \mathrm{mg} / 1000$ & $87,2 \mathrm{mg} / 1000$ & 0,03 & Non significative \\
\hline
\end{tabular}

* Pour 98 degrés de libertê la différence des moyennes est significative entre le groupe têmoin et celui des animaux parasítês aux niveaux. $t 0,05: 1,99$ et $t 0,01: 2,63$.

tabolique "), compensée par une synthèse accrue de gamma globulines.

\section{Bilirubine totale}

La variation de ce constituant est considérée comme non significative par $\mathrm{O}$. MOLINARI (20) et A. A. VASIL'EV (28). S. K. DWIDEDI considère son augmentation comme significative à $\mathrm{P}=0,01(8)$.

La valeur moyenne relevée dans notre étude est de $5,3 \mathrm{mg} / 1000 \mathrm{chez}$ les zébus parasités contre $7,1 \mathrm{mg} / 1000 \mathrm{chez}$ les témoins (variation hautement significative). Cette diminution paraît aberrante compte tenu de l'atteinte hépatique. Aussi est-ce à la variation de ce constituant que nous attachons le plus grand intérêt pour le diagnostic de la fasciolose. De plus, au cours d'une étude similaire sur la tuberculose, la moyenne relevée chez les sujets tuberculeux était de $7,12 \mathrm{mg} / 1000$ contre $7,19 \mathrm{mg} / 1000$ chez les témoins $(t=0,17)$. L'interférence est donc impossible entre ces deux maladies chroniques, ce qui renforce la spécificité de l'analyse. A partir du seuil de 4,1 mg/1000 il s'agit, une fois sur deux, d'un animal exempt de fasciolose.

\section{Transaminases T.G.P.}

Cette analyse a été peu pratiquée dans la fasciolose bovine. J. HANKIEWICZ (14) ne trouve pas de variations significatives des transaminases lors de l'infestation par "Fasciola hepatica ". Dans notre étude, si la transaminase glutamique-oxaloacétique ne varie pas significativement, la transaminase glutamique-pyruvique a par contre une activité diminuée de façon hautement significative chez les sujets parasités. Cette variation aberrante est confirmée et expliquée par celle de la bilirubine. Elle pourrait être utilisée, au même titre qu'elle, comme épreuve de diagnostic quoique de façon moins pratique. 


\section{Protéines sériques}

Leur taux augmente de façon significative, ce qui correspond aux résultats des recherches dans l'infestation par "Fasciola hepatica" (2, 10, 25) sauf celles de J. KONRAD (18).

Comme dans le cas des variations du protéinogramme il s'agit d'une modification non spécifique, notée dans des états infectieux variés. Aussi n'est-elle pas utilisable dans un but diagnostique.

\section{Autres constituants}

Leur variation n'est pas significative, et n'a pas été étudiée par d'autres auteurs sauf celle du calcium $(9,18)$ qu'ils trouvent également sans signification.

\section{SUMMARY}

The change in the composition of the blood

in bovine fasciolosis (Fasciola gigantica)

Among 12 biochemical parameters of 50 zebu cattle affected with acute fasciolosis, 6 are significantly, or highly significantly, different from 50 controls: albumin, bilirubin, G.P. transaminase, gamma and beta globulines and total serum proteine content. Bilirubin and G. P. transaminase changes seem connected with Fasciola gigantica infection.

\section{RESUMEN}

\section{Modificaciones de algunos constituyentes bioquímicos de la sangre en cebues naturalmente infestados por Fasciola gigantica}

El dosaje de 12 constituyentes bioquímicos de la sangre de 50 cebues atacados por fascioliasis grave demuestra que la tasa de 6 de ellos varia de modo significativo o muy signifícativo con relación a la observada en 50 animales testigos.

Se tratan de las tasas de albumina, de bilirrubina, de transaminasa glutamica-piruvica, de gama-globulinas, de beta-globulinas y de proteinas sericas. Son las variaciones de las tasas de bilirrubina y de transaminasa glutamica-piruvica que parecen muy ligadas con la fascioliasis y pueden confirmar el diagnóstico de esta parasitosis.

\section{BIBLIOGRAPHIE}

1. BALIAN (B.). Ricerche ematologiche e biochimiche in bovini normali ed affetti da distomatosi epatica. Nuova Vet., 1940, 18 : 134-142.

2. BALIAN (B.). Ricerche ematologiche e biochimiche in ovini normali ed affetti da distomatosi epatica. Nuova Vet., $1940,18: 154-160$.

3. BAYSU (N.), TIGIN (Y.) et GURALP (N.). Fasciola gigantica ile enfekte edilmis keyunlarin serumunda specifik karaciuger enzimlerinin diagnoz yönünden önemi ve bu enfeksiyon dolayisi ile kan tablesunda maydama gelen degisiklikler 2. Veteriner Fakulltesi dergisi, 1971, 18 (1) : 97110.

4. BLANCOU (J.), BOUCHET (A.) et DAYNES (P.). Etude sur l'allergie, les anticorps précipitants et les anticorps fixant le complément au cours de l'infestation par Fasciola gigantica. Rev. Elev. Méd. vét. Pays trop., 1971, 24 (3) : 373-379.

5. BOUCHET (A.), DAYNES (P.) et GAULIER (E.). Etude de quelques modifications sanguines chez les bovins au cours d'une infestation de moyenne importance par Fasciola gigantica. Influence du traitement.

Rapport I.E.M.V.T., 1971. Archives I.E.M.V.T.

6. CHO (T. H.) et KIM (D.W.). Serum quinine oxidase activity of koreas cattle with hepatic fascioliasis, Res. Rep. off. rur. Dev. Scuron, Korea, 1968, 11 (5) : 83-89.

7. DREPPER (K.) et KEDENBURG (C.P.). Activity of some liver specific enzymes in the serum of sheep experimentally infected with Fasciola hepatica. Tierärztl Umsch., 1967, 22 : 396-397.

8. DWIDEDI (S. K.), JOSHI (H. C.) et SHIUNANI (G. A.). Evaluation of liver function tests in Fasciola infection in cattle and buffaloes. Indian J. anim. Hith., 1972, 11 (1): 81-84.

9. ENIGK (K.), FEDER (H.), DEY-HAZRA (A.) et WEINGARTNER (E.). Mineralstoffgehalt des Blutplasmas, der Erythrocyton und der Leber Wänrend der Präpatenz der Fasciolose beim Rind. Zentbl. Vet. med., 1972, 19 B (3) : 238257.

10. FALASCHINI (A.) et MANTOVANI (G.). Osservazioni sul comportamento delle proteine del siero di sangue in bovini da macello colpiti da lesioni parassitarie a sede epatica. Ann. Fac. Med. Vet. Torino 1954, 4 : 213-217.

11. FLEURY (P.), COURTOIS (J. E.) et LECLERC (M.). Fiches techniques de chimie biologique. Paris, VEGA, 1965.

12. GENCHI (C.) et LOCATELLI (A.), Sorbitol dehydrogenase and aspartate transaminase in 
cattle affected with fascioliasis. Atti. Soc. Ital. Sci. Vet., 1969, 22 (1968): 565-567.

13. GORYACHEV (V.E.). Changes in carbohydrate-protein complexes in the blood of sheep with fascioliasis, and their clinical significance. Mater Konf. vses Obshch Gel'mint, Moskova, 1967, 2 : 96-102.

14. HANKIEWICZ (J.), HANKIEWICZ (K.). Serum (glutamic oxalic and glutamic pynuvic) transaminase activity in slaughtered cattle infected with fascioliasis. Wien tierartzl. Wschr., 1964, $\mathbf{5 1}$ : 145-151 (G.c).

15. KAMALYAN (G. V.), ABRAMYAN (E.G.), MNATSAKANYAN (A. A.) et BUNIATYAN (L. O.). The free amino-acid content and the transaminase activity of the serum, liver and kidneys in experimental fascioliasis in sheep and rabbits. Biol. Zh. Arm., 1969, 22 (6) : 92-95.

16. KEARNEY (A.), CONNOLLY (J. F.) et DOWNEY (N. E.). Serum transaminase levels in treated Fasciola hepatica infected sheep. Vet. Rec., 1967, 81 (6): 134-139.

17. KONA (E.). Elektroforetické vysétrovanie bielkovinovych frakcú krvného séra a peritonealného i perikardialneho exsudatú u oviec, chorych na fasciololózu. Vet. Cas., 1957, 6: 146150.

18. KONRAD (J.). The biochemical picture in bovine hepatic cirrhosis due to Fascioliasis. Tierátzl. Umsch., 1968, 23: 239-372, 375-376.

19. LOISELEUR (J.) et Collab. Techniques de Laboratoire. Paris, Masson, 1953.
20. MOLINARI (P.) et VALCARENCHI (G.). Indagini funzionali del fegatonella distomatosi dei bovini. Veterinaria, Milano, 1959, 8: 71-74.

21. MULLIGAN (W.). The effect of helminthic infection on the protein metabolism of the host. Proc. Nutr. Soc., 1972, 31 (1): 47-51.

22. PINKIEWICZ (E.) et MADEJ (E.). Changes in the peripheral blood of $\mathrm{Ca}, \mathrm{P}, \mathrm{K} . \mathrm{Na}, \mathrm{Mg}$ and AP in the course of experimental fascioliasis in sheep. Acta parasit. polon, 1967, 15 (30) : 225229.

23. REITMAN (S.) et FRANKEL (F.). Am. J. Chim. Path., 1957, 28 : 56.

24. SINCLAIR (K. B.). Serum calcium and magnesium levels in sheep infected with Fasciola hepatica. Vet. Rec., 1960, 72: 506.

25. SINCLAIR (K. B.). Observations on the clinical pathology of ovine fascioliasis. Brit. vet. J., 1962, 118 : 37-53.

26. TOMICKI (Z.) et MALINOWSKA (A.). Próba bromsulftaleinowa (B.S.F. clearance) w motylicy watrobowej u bydla. Med. Vet., 1961, 17: 591594.

27. TIGIN (Y.), BAYSU (N.) et GURALP (N.). Fasciola gigantica ile enfekte edilmis loyunlarin serumunda spesifik karaciuger enzimlerinin diagnoz yönünden önemi ve bu enfeksiyon dolayisi ile kan tablosunda meydana gelen gegisilkikler 1. Veteriner Fakültesi Dergisi, 1971, 18 (1): 8196.

28. VASIL'EV (A. A.). Study of some parameters of metabolism in cattle with spontaneous fascioliasis, before and after anthelminthic treatment. Sbornik Rabot po gel' mintologü (Vses. Inst. gel' mint), 1967, 13 : 253-268. 